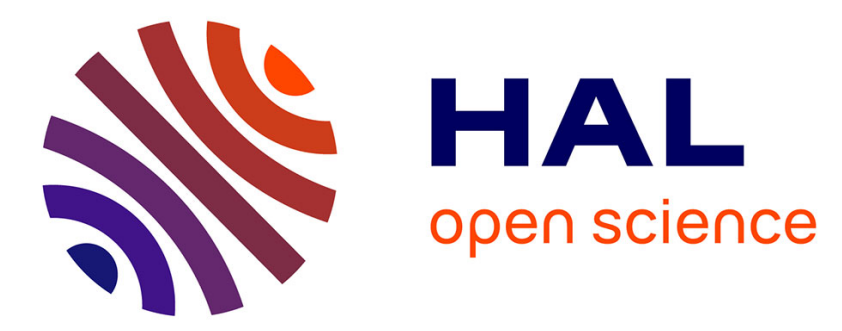

\title{
Dégradation progressive du milieu par le surpâturage et le déboisement et conséquences hydrologiques : étude de cas dans la Sierra Madré occidentale (Mexique)
}

\author{
David Viramontes, Luc Descroix
}

\section{- To cite this version:}

David Viramontes, Luc Descroix. Dégradation progressive du milieu par le surpâturage et le déboisement et conséquences hydrologiques: étude de cas dans la Sierra Madré occidentale (Mexique). Les dossiers de la Revue de Géographie Alpine, 2002, 2, pp.27-41. 10.3406/rga.2000.2989 ird-02157739

\author{
HAL Id: ird-02157739 \\ https://hal.ird.fr/ird-02157739
}

Submitted on 17 Jun 2019

HAL is a multi-disciplinary open access archive for the deposit and dissemination of scientific research documents, whether they are published or not. The documents may come from teaching and research institutions in France or abroad, or from public or private research centers.
L'archive ouverte pluridisciplinaire HAL, est destinée au dépôt et à la diffusion de documents scientifiques de niveau recherche, publiés ou non, émanant des établissements d'enseignement et de recherche français ou étrangers, des laboratoires publics ou privés. 


\section{Dégradation progressive du milieu par le surpâturage et le déboisement et conséquences hydrologiques Étude de cas dans la Sierra Madre occidentale (Mexique)}

\section{David Viramontes}

Laboratoire TEO - UMR CNRS 5038 - Université J. Fourier, IGA - 17 rue M. Gignoux F38031 Grenoble cedex viram@iga.ujf-grenoble.fr

\section{Luc Descroix}

LTHE-IRD - BP 5338041 Grenoble cedex 9 Grenoble - descroix@hmg.inpg. fr

\section{Origine de l'étude}

L'évolution du milieu peut se refléter sur l'évolution du régime des cours d'eau. I.es impacts des changements du milieu à une échelle de temps rapide et sur une grande superficie (construction de barrages, déviation des cours d'eau pour l'irrigation, nouvelles zones mises en culture, modifications du modelé dues aux séismes, erc.) peuvent rapidement se traduire par des modifications du régime des écoulements.

Cependant, les modifications progressives dans le temps et diffuses dans l'espace (surpâturage, déforestation, urbanisation, etc.), n'ont pas toujours de répercussions visibles sur les régimes des cours d'eau sauf si ces changements sont déjà très marqués dans l'espace.

Des travaux effectués sur des bassins versants expérimentaux comparables et sur des tendances statistiques sont souvent nécessaires pour détecter des modifications des régimes des écoulements. Néanmoins, la plupart de ces études ont mené à la recherche d'effers quanritatifs sur le bilan hydrique. C'est-à-dire que l'intérêt principal est de savoir si un site modifié physiquement produit plus ou moins d'écoulement.

Par ailleurs, le rôle hydrologique d'une formation végétale ou d'un paysage déterminé dépend de la combinaison de conditions climatiques et pédologiques. Ainsi, plus les sols ont une réserve en eau importante, et plus le climat présente des périodes de surplus et de déficit en eau dans l'année, plus les changements de cetre formation ont des répercussions sur le bilan d'écoulement annuel (Cosandey, 1995). Ainsi, une forêt méditerranéenne joue un rôle plus important qu'une prairie continentale, et encore plus qu'une zone aride. 
Dans le cas qui nous intéresse ici, la Sierra Madre (Occidentale a subi une dégradation progressive (surpâturage et déforestation) depuis plusieurs décennies. Elle présente un climat subtropical avec une longue saison sèche dans l'année ( 8 à 9 mois), er des sols peu épais (Phacozems et I ithosols surtout). Nous nous sommes intéressés à la recherche de ces changements quantitatifs sur le bilan hydrique, mais aussi aux modifications du comportement des écoulements.

\section{Problématique de la Sierra Madre occidentale}

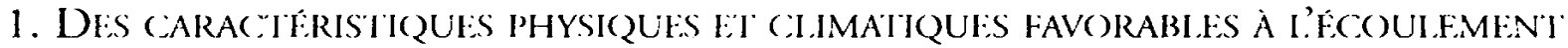

La Sierra Madre Occidentale est une chaîne montagneuse massive culminant en de nombreux points à plus de $3000 \mathrm{~m}$ d'altitude. Elle est parallèle à la côte du Coolfe de Californie et à l'Océan Pacifique depuis la frontière des États Unis vers le Sud, sur une longueur de plus de $2000 \mathrm{~km}$. Constituée surtout de roches acides volcaniques du Tertiaire, elle présente la couverture ignimbritique continue la plus grande du monde (Demant et Robin, 1975, et McI Jowell et Clabaugh, 1979).

D’une manière générale, les sols sont de peu à moyennement profonds (en général de 10 à $50 \mathrm{~cm}$ ) et ont des capacités de stockage d'eau faibles $(119$ à $125 \mathrm{~mm}$ ). Les très rares sols profonds (jusqu’à $100 \mathrm{~cm}$ ) ayant des capacités de stockage d'eau importantes (plus de $300 \mathrm{~mm}$ ) se trouvent dans le bas du versant (Viramontes 1995).

Selon la latitude, le climat de la Sierra Madre Occidentale peut être rattaché à l'ensemble des climats subtropicaux secs; cependant, la proximité du Pacifique et de la Convergence Intertropicale, donne à cette zone un régime pluviométrique tropical (saison des pluies d'été qui représentent plus de $75 \%$ du total annuel, et plus de six mois secs) (Nouvelot et Iescroix, 1996). L'altitude de la zone lui confere des éléments propres des climats de montagne (gel hivernal, amplitudes thermiques fortes, pluviométrice bien plus abondante qu'en plaine). Le déplacement de masses d'air est typiquement subtropical mais présente de grandes variabilités internes dues essentiellement au relief et aux variations d'altitude. Le gradient thermique moyen est de $0.6 \% 100 \mathrm{~m}$, celui de la pluviométric est de $32 \mathrm{~mm} / 100 \mathrm{~m}$. I.e tableau 1 donne quelques valeurs climatiques caractéristiques.

\begin{tabular}{|l|c|c|c|c|}
\hline Station & $\begin{array}{l}\text { Altitude } \\
(\mathrm{m})\end{array}$ & $\begin{array}{l}\text { Pluviométrie annuelle } \\
\text { moyenne }(1960-90) \mathrm{mm}\end{array}$ & $\begin{array}{l}\text { Cv de la } \\
\text { pluviométrie }\end{array}$ & $\begin{array}{l}\text { Température } \\
\text { moyenne }{ }^{\circ} \mathrm{C}\end{array}$ \\
\hline Tepehuanes & 1800 & 463 & $\mathbf{0 . 1 7}$ & 16 \\
$\begin{array}{l}\text { La Cienega de Escobar } \\
\text { El Tarahumar }\end{array}$ & 2100 & 612 & $\mathbf{0 . 2 1}$ & 14 \\
El Cuije (désert de Chihuahua) & 2850 & 971 & $\mathbf{0 . 1 7}$ & 10.5 \\
pour comparaison & 1100 & 190 & $\mathbf{0 . 4 2}$ & 21 \\
\hline
\end{tabular}

CV $=$ coefficient de variation 
D'autre part, la station I.a Cienega de Escobar connaît environ 100 jours de gel par an.

L'ensemble de caractéristiques topographiques et climatiques donne à cette zone des coefficients d'écoulement moyens de l'ordre de 15 à $17 \%$, ce qui permet aux cours d'eau d'atteindre l'Altiplano Nord Mexicain avec suffisamment d'eau pour y rendre possible l'irrigation.

La Sierra Madre Occidentale constitue donc, un véritable " château d'eau " pour les zones voisines plus basses semi-arides et arides. Ces zones sèches ont profité des écoulements pour établir des zones agricoles importantes et des activités agro-industrielles. La vallée de Sonora, les vallées de l'État de Chihuahua (Delicias et Jimenez.), et la Comarca lagunera (fig. 1) en sont des exemples.

\section{UN RISQUE IDE I FESEQQUHIBRE I)U BII.AN IHYDROIOCGIQUE:}

\section{a. La récente évolution de l'espace}

L'histoire du Nord du Mexique ressemble plus à celle de l'Ouest américain qu'à celle du Centre et du Sud du pays, où de grandes civilisations ont bien marqué le paysage.

Jusqu'au XIX siècle cette zone d'étude n'était habitée que par des indiens Tarahumaras et 'lepehuanos, vivant dans de petits villages disséminés dans la Sierra. Les seules exceptions étaient les sites miniers autour desquels sont apparues des villes coloniales, existant encore aujourd'hui, presque isolées du pays (Tepehuanes, Santa Maria del Oro, (juanacevi, etc.). À la fin de ce même siècle, sous la forme de grandes Haciendas, il y a eu une grande colonisation par des populations étrangères à la région. I a région devient donc, une zone d'élevage extensif avec une "logique d'exploitation minière " des ressources (on détruit le capital sans veiller à son renouvellement).

Après la période de crises de la Révolution de 1910 et à la suite de la Réforme Agraire de 1936, sont apparus les "ejidos", communautés paysannes ayant un système de production collectif. Entre 1948 et 1970 les ejidos se sont installés dans notre zone d'étude, et ils ont conservé la même tradition d'élevage et la même logique de production que les haciendas dissoutes.

Au cours des dernières années un nouveau phénomène se présente. I a popularion de la Sierra avait toujours augmenté jusqu'aux années 70-80 ; par la suire, elle a commencé à décroître, malgré un taux de natalité élevé. En effet, une très forte proportion de jeunes choisit de partir, le plus souvent aux États Unis. La plupart d'entre cux ne revient plus, ou bien, seulement pour les vacances. De ce fait, la population des ejidos diminue à une vitesse très rapide. Cependant, l'activité agro-pastorale se maintient, voire progresse, du fait des gains de productivité. I es habitants qui restent dans les communautés reçoivent une aide économique de ceux qui sont partis. Ils gardent leurs troupeaux, qui sont, pour ainsi dire, subventionnés par leurs parents vivant au Texas, au Nevada, en (alifornic ou dans l'Illinois (principales destinations des migrants). 


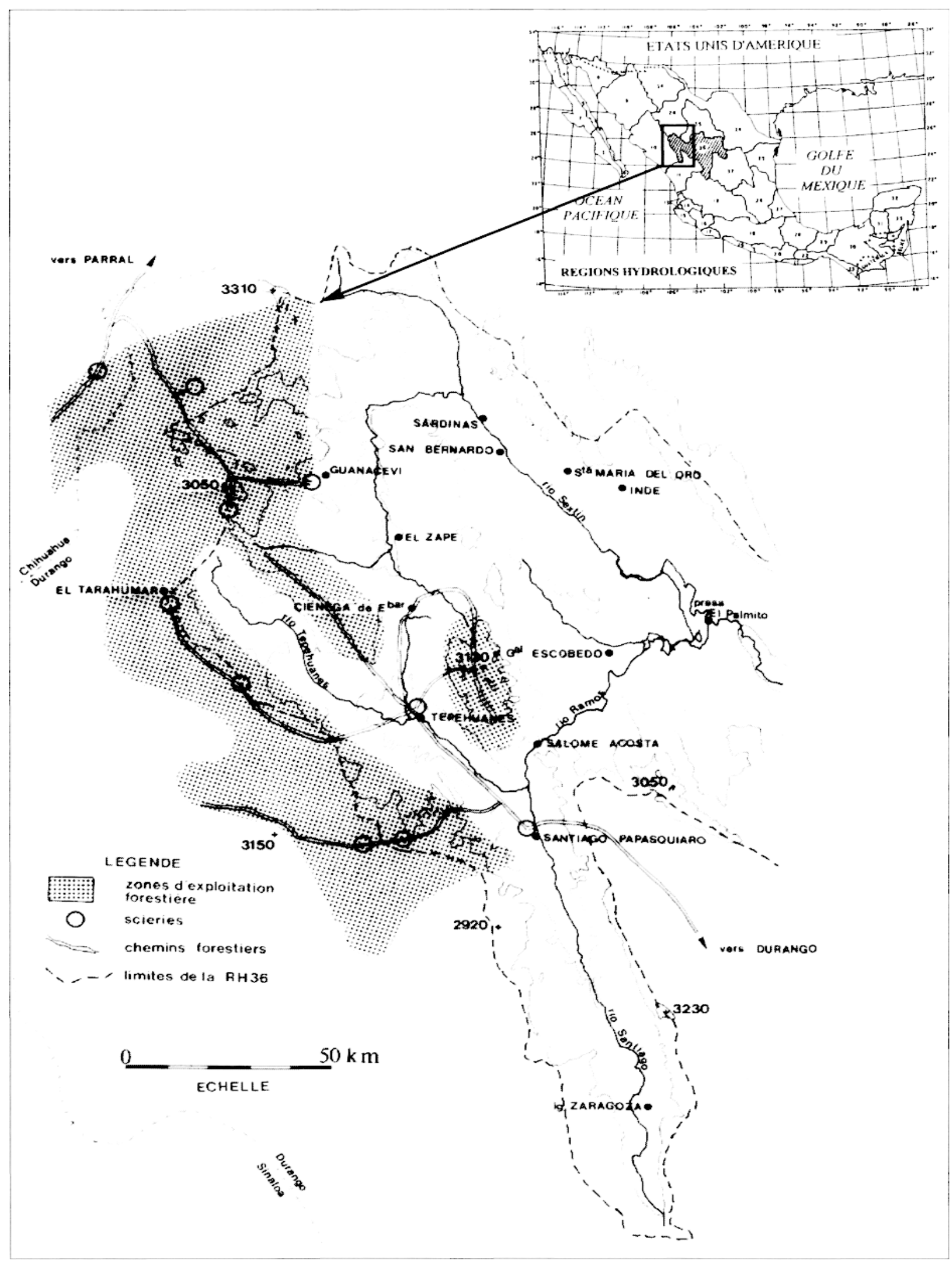

(sont figurées les limites des états intéressés, à savoir Durango, Sinaloa et Chihuahua)

Fig. 1 : Localisation du Haut bassin du Rio Nazas 
Actuellement, le texte réglementaire de référence est la Réforme Agraire de 1992 : il donne aux paysans leur titre de propriété et permet donc de vendre ou d'acheter les terres qu'ils exploitaient auparavant en étant simplement usufruitiers. Dans la Sierra quand les terres se libèrent il y a toujours d'autres "ejidatarios" qui les prennent en main. Donc, on se dirige vers l'agrandissement de la superficie des exploitations par habitant avec plus de financement pour l'exploitation. Selon cette logique, la production augmente, mais la pression sur le milicu se maintient malgré la très rapide dépopulation

\section{b. Un milieu marqué par des traces de surexploitation}

\section{b.1. Le surpâturage}

Malgré un système d'exploitation rustique et peu rentable, l'élevage extensif est l'activité économique principale et traditionnelle de cette région. Pour les éleveurs, la seule manière de gagner davantage est d'augmenter leur nombre de têtes de bétail.

L'étude sur le pâturage présentée par Barral et Anaya (1995) pour 4 ejidos de la zone d'étude estime que le volume de biomasse disponible est faible, seulement 130 à $2070 \mathrm{~kg} / \mathrm{ha}$ avec une moyenne de $723 \mathrm{~kg} / \mathrm{ha}$. En considérant les caractéristiques du bétail utilisé, une Unité Bovine (UB) consomme de manière journalière trois pour cent de son poids en matière sèche, et avec un poids moyen normal de $450 \mathrm{~kg}$, la consommation journalière nécessaire de chaque UB est de $13,5 \mathrm{~kg}$, c'est-à-dire près de $5000 \mathrm{~kg} / \mathrm{an}$.

I) fait de la faible productivité des pâturages, on a dû utiliser ici des ha/UB et non l'inverse, pour ne pas avoir à fractionner les UB. D'après ces données, la charge moyenne de pâturage théorique souhaitable serait de 13,6 ha/UB, avec des valeurs de 10,5 ha/UB par exemple pour l'ejido la Posta de Jihuites et 19,4 ha/UB pour Boleras. Cependant, la charge de bétail est quatre fois supérieure : elle est de 2,25 ha/UB à la Posta de Jihuites et 3,95 ha/UB à Boleras. I)e surcroît, la surcharge de bétail est répartic d'une façon très hétérogène. Près des sources d'eau et dans les villages le surpâturage est encore plus marqué.

I.es conséquences en sont évidentes sur le paysage. I ans certaines zones l'augmentation des espèces ligneuses (principalement des pins de petite taille) et la diminution des graminées sur des sols caillouteux dégradés, fait penser à une " dégradation verte des prairies" (Gentry, 1957, Boutrais, 1994, et Viramontes, 1995); le maintien des pâturages est gêné par l'envahissement des pins. L'autre preuve de surpâturage est la formation de terrassettes sur les versants (Poulenard et al, 1995). (é phénomène est connu dans les Alpes et les Andes (Serrate, 1978) comme conséquence des cheminements préférentiels du bétail sur les courbes de niveau (photos 1 et 2).

\section{b.2. Le déboisement}

I a forme d'exploitation forestière est extensive et sélective, elle ne montre pas donc une grande superficie déboisée. Les coupes forestières ne laissent pas des superficies ouvertes et même, les exploitants sont obligés de planter des perits arbres pour la nouvelle 
génération. Cependant, l'exploitation forestière illégale et la forte demande de bois pour l'industrie font que les entorses à cette obligation de reboiser sont nombreuses. D'après le travail réalisé par Rodriguez (1997), la déforestation est rapide sur les Unités d'Administration Forestières (UAF) de Tepehuanes et Guanacevi correspondant à cette zone d'étude.

I.es images satellites prises en 1972 et 1992 montrent une diminution de la densité de la couverture végétale (tableau 2). La superficie couverte par la forêt diminue et la formation de savane ouverte et les zones sans végétation gagnent du terrain. Les pâturages non arborés se maintiennent (ils gagnent sur la forêt d'une part et sont envahis par les jeunes arbres par ailleurs).

\begin{tabular}{|c|c|c|c|}
\hline Formation végétale & $1972\left(\mathrm{Km}^{2}\right)$ & $1992\left(\mathrm{Km}^{2}\right)$ & Évolution en \% \\
\hline $\begin{array}{l}\text { Forêt dense } \\
\text { Forêt intermédiaire } \\
\text { Forêt claire } \\
\text { Formation de type "savane d'altitude " } \\
\text { Zones de pâturage } \\
\text { Zones sans végétation }\end{array}$ & $\begin{array}{r}400 \\
1537 \\
1967 \\
1417 \\
1591 \\
758\end{array}$ & $\begin{array}{r}155 \\
672 \\
1203 \\
1805 \\
1614 \\
905\end{array}$ & $\begin{array}{l}-62 \% \\
-54 \% \\
-39 \% \\
+27 \% \\
+0.2 \% \\
+19 \%\end{array}$ \\
\hline
\end{tabular}

Tableau 2 : Formations végétales de la Sierra Madre Occidentale en 1972 et 1992, à partir des images Landsat

\section{c. Les observations à l'échelle de la parcelle.}

Différents travaux de mesures quantitatives faits depuis 1994 sur des parcelles d'érosion et bassins versants expérimentaux ont permis de faire un certain nombre d'observations; on résume par la suite méthodes et résultats (Descroix et Nouvelot, 1997).

Des parcelles de mesure ont été installées en zone de forêt et en zone de pâturages : 13 parcelles de $50 \mathrm{~m}^{2}, 3$ parcelles de $10 \mathrm{~m}^{2}$ et 42 parcelles de $1 \mathrm{~m}^{2}$ ont été installées pour connaître l'impact des changements observés (surpâturage, déboisement) dans le plus grand nombre de configurations (pente, lithologie, types de sols et d'états de surface, clôture éventuelle, taille de la parcelle, types d'arbres sous forêt et savane). D'autre part, 5 bassins-versants expérimentaux ont été équipés. Sur toutes les parcelles et en de nombreux points des bassins versants, des mesures de conductivité hydraulique à saturation ont été réalisćes (par infiltromètre à disque). Les mesures ont duré 2 à 4 ans suivant les secteurs. Quelques mesures ont été résumées dans les tableaux 3 et 4.

\begin{tabular}{|l|c|c|c|}
\hline Parcelles de $50 \mathrm{~m}^{2}$ & sans arbre & sans arbre mais avec litière & sous l'arbre \\
\hline Coefficient de ruissellement $\%$ & 23 & 8.5 & 2.8 \\
Érosion en $\mathrm{g} / \mathrm{m}^{2}$ & 133 & 30.5 & 1.11 \\
\hline
\end{tabular}

Tableau 3 : Influence de la coupe des arbres sur le ruissellement et l'érosion en forêt 


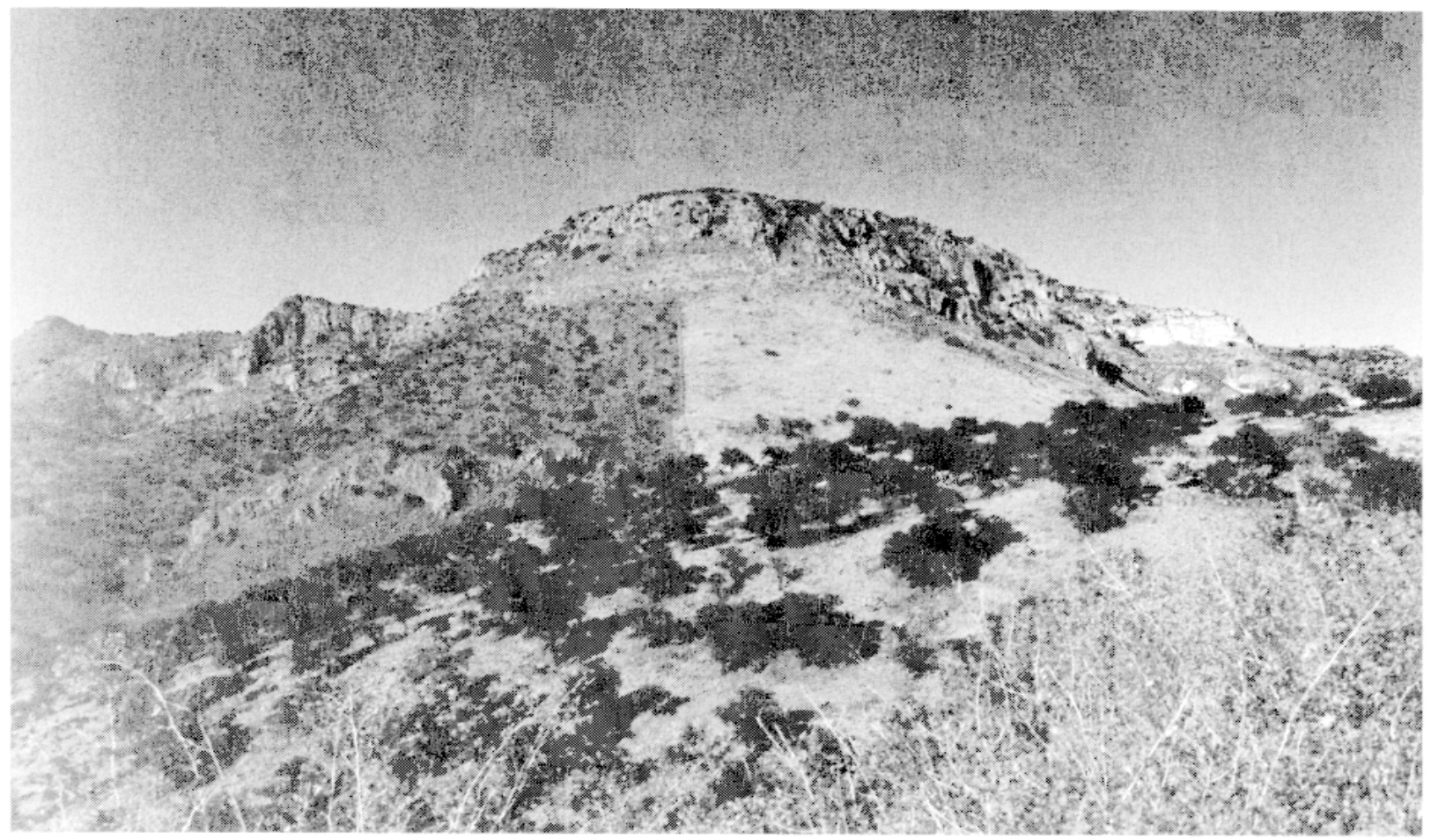

Photo 1. Conséquences du surpâturage : à gauche de la clôture, secteur surpâturé, sombre, car sans graminées et envahi par des ligneux (genévriers)

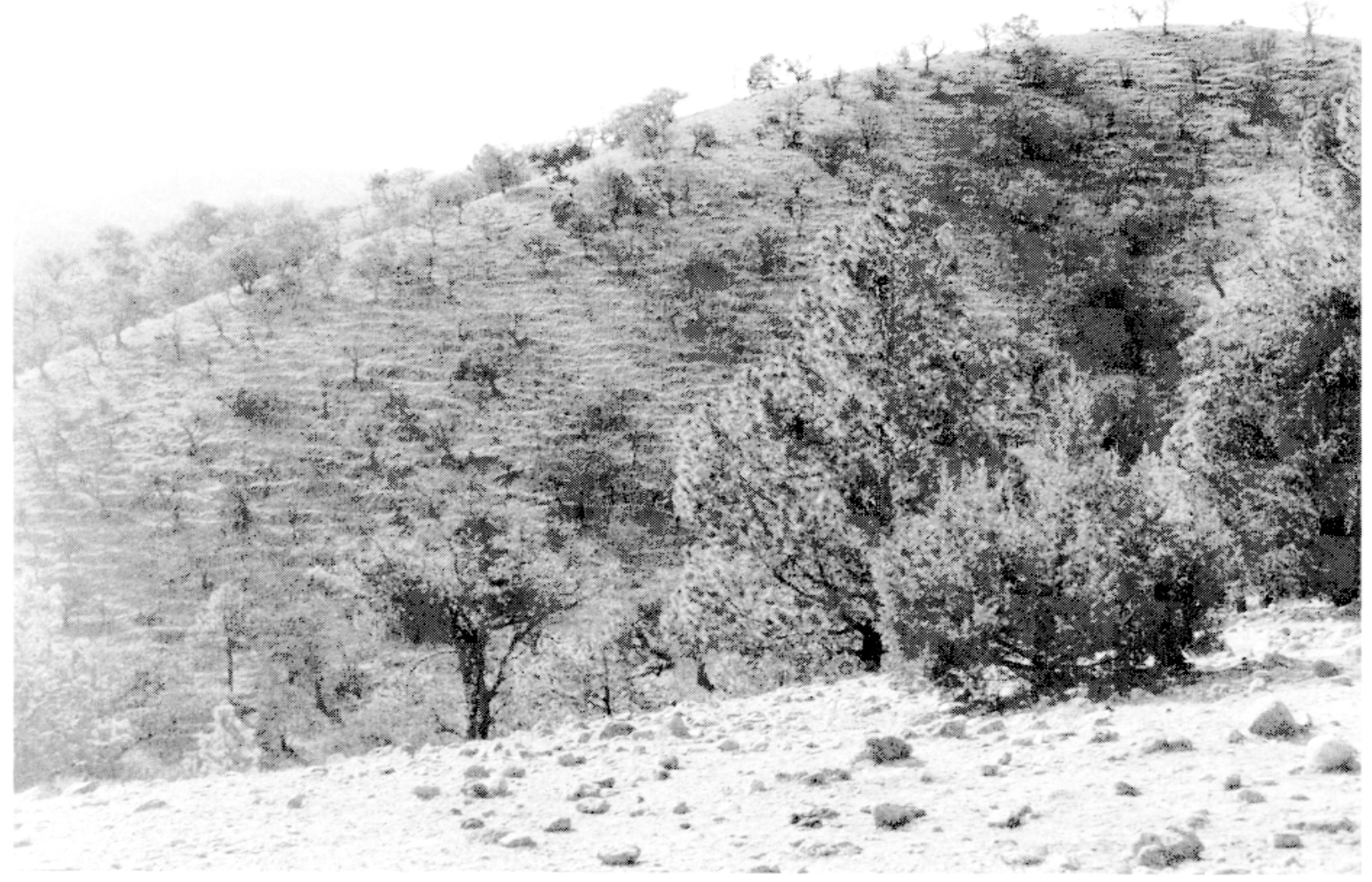

Photo 2. Conséquences du surpâturage : au premier plan, les graminées sont presque inexistantes, les cailloux de plus en plus nombreux ; deuxième plan, sur les pentes supérieures à $15^{\circ}$, apparition de terrassettes dues au piétinement du bétail. 


\begin{tabular}{|l|c|c|}
\hline Micro-parcelles de $1 \mathrm{~m}^{2}$ & Piétinées (8 parc.) & non-piétinées (8 parc.) \\
\hline Coefficient de ruissellement en $\%$ & 43 & 7 \\
Érosion en $\mathrm{g} / \mathrm{m}^{2}$ & 90 & 1.31 \\
Densité apparente en $\mathrm{g} / \mathrm{cm}^{3}$ & 1.38 & 0.008 \\
Conductivité hydraulique en mm/s & 0.0035 & 7.3 \\
\hline
\end{tabular}

Tableau 4 : Influence du piétinement du bétail sur le ruissellement et l'érosion en zone de pâturages

1. On note un encroûtement des sols sur les secteurs les moins pentus, du fait du piétinement du bétail, de la dégradation de la végétation, et de l'exagération du splash qui en découle ; ces sols encroûtés ont des coefficients de ruissellement et des taux d'érosion bien plus élevés que les sols non transformés;

2. Les zones les plus pentues (plus de $25 \%$ ) finissent par être pavées de pierres du fait de l'érosion hydrique, qui emporte en premier lieu les particules fines; à terme, ce pavage protège le sol, ce qui tend à faciliter l'infiltration de l'eau et, en dispersant l'énergie cinétique, à limiter l'effet splash et l'érosion ;

3. En forêt comme en savane, la coupe des arbres induit un effet splash accru (par disparition de la litière, d'où rejaillissement, fermeture des pores, et encroûtement), ce qui a pour effet d'augmenter le ruissellement et l'érosion, et de diminuer la conductivité hydraulique du sol à saturation ;

4. On observe aussi des valeurs plus faibles de conductivités hydrauliques à saturation dans les sols encroûtés et surpâturés.

5. Ruissellement et érosion se font de manière aréolaire bien plus que linéaire ; les ravines sont de grande taille (plusieurs centaines de mètres, jusqu'à deux ou trois kilomètres) et profondes (de 2 à 10 mètres), mais sont rares et circonscrites aux zones peu pentues et cultivées, aux sols profonds ;

6. Sous forêt, la couverture des chênes est plus efficace que celle des pins contre l'érosion et la formation du ruissellement ;

7. En zone de savane arborée d'altitude, on note une invasion des pâturages par les pins, espèce conquérante qui remplace les chênes mais entraîne une dégradation de la qualité des herbages (concurrence avec les graminées et acidification des pâturages).

Un premier bilan à l'échelle des parcelles et petits bassins se traduit par une augmentation des coefficients d'écoulement (et des valeurs de pertes en sol) comme conséquences du surpâturage et du déboisement ; mais nous ne pouvons pas généraliser à l'échelle des grands bassins-versants. La prise en compte de superficies beaucoup plus vastes et la grande variabilité de l'espace montagnard de la zone d'étude pourraient compenser cet effet. Les travaux sur le thème de l'influence des modifications du milieu sur les régimes climatiques ou hydrologiques concluent en général sur l'importance du stockage de l'eau dans l'ensemble sol-végétation (Bosch et Hewlett, 1982 ; Fritsch, 1990 ; Cosandey, 1995). 


\section{Conclusion de la problématique}

I a Sierra Madre Occidentale a subi une évolution progressive de dégradation du milieu comme conséquence des activités humaines. Elle représente donc une zone fragilisée où les changements climatiques et environnementaux pourraient avoir des répercussions locales et régionales. Il est important pour cette zone de montagne comme pour les zones aval de connaître les risques causés par cette évolution du milieu.

Au niveau expérimental, les résultats résumés ci-dessus, observés sur parcelle et petit bassin versant nous ont montré l'effet direct des activités humaines sur la végétation, les sols et les eaux superficielles, mais il n'est pas question de généraliser les résultats pour toute la zone. Dans le prochain chapitre, on essaiera de mettre en évidence les conséquences déjà perceptibles ou envisageables à une échelle plus perite sur les écoulements fluviaux.

\section{Mémonomogit:}

Le site particulier d'étude est le haut bassin du Rio Nazas qui est localisé au coeur de la Sierra Madre Occidentale, dans l'État de Durango. S'écoulant vers le côté continental de la Sierra, il représente la principale zone d'approvisionnement en eau d'une des régions endoréiques du pays (la Région Hydrologique 36 avec $92000 \mathrm{~km}^{2}$, constituée des bassins versants des Rio Nazas et Aguanaval). Il apporte 87,5\% des écoulements superficiels qui arrivent dans le fond de la dépression, au périmètre irrigué de la Comarca Lagunera ( 160000 ha et près d'un million d'habitants dans les villes de la Laguna). On utilise les données hydro-pluviométriques des deux sous-bassins naturels (non influencés par des ouvrages) du haut Nazas : ceux du Rio Sextin et du Rio Ramos.

Nous avons utilisé les données de pluies des 42 stations météorologiques localisées dans la zone d'étude, et suivies par les services régionaux de la "Comisión Nacional del Agua " (CNA) du Mexique. I a densité du réséau météorologique est faible, mais les stations sont assez bien réparties dans l'espace et en altitude; cela confere à l'ensemble du réseau une bonne représentativité spatiale.

Les calculs des lames d'eau précipitées qui ont permis de calculer les coefficients d'écoulement ont été faits par interpolation en mode krigeage.

\section{Les tendances statistiques des coefficients d'écoulement}

Dans un premier temps, on a cherché des tendances statistiques sur les changements quantitatifs dans la relation pluie/débit. (On peut constater (fig. 2) que l'évolution temporelle de la pluviométrie ne montre aucune tendance visible. La diminution sensible des coefficients d'écoulement et des lames écoulées depuis 1994 inclus est due avant tout à une succession d'années déficitaires, sauf 1996 qui est légèrement excédentaire, et non à une tendance. 


\section{La décomposition des hydrogrammes}

Une des méthodes les plus connues pour différencier les écoulements de base des écoulements de crue d'une série de données de débits journaliers est "la méthode de l'indice d'écoulement de base ", basée sur l'algorithme de (iustard (1989) tel que proposée par Humbert et Kaden (1994). Cette méthode part de l'hypothèse suivante : "In admettant que les effers climariques ont été éliminés, toute modification sensible des paramètres du milieu doit se répercuter sur l'évolution de la proportion respective du débit de base $(Q B)$ et du débit de crue $(Q C)$ ".

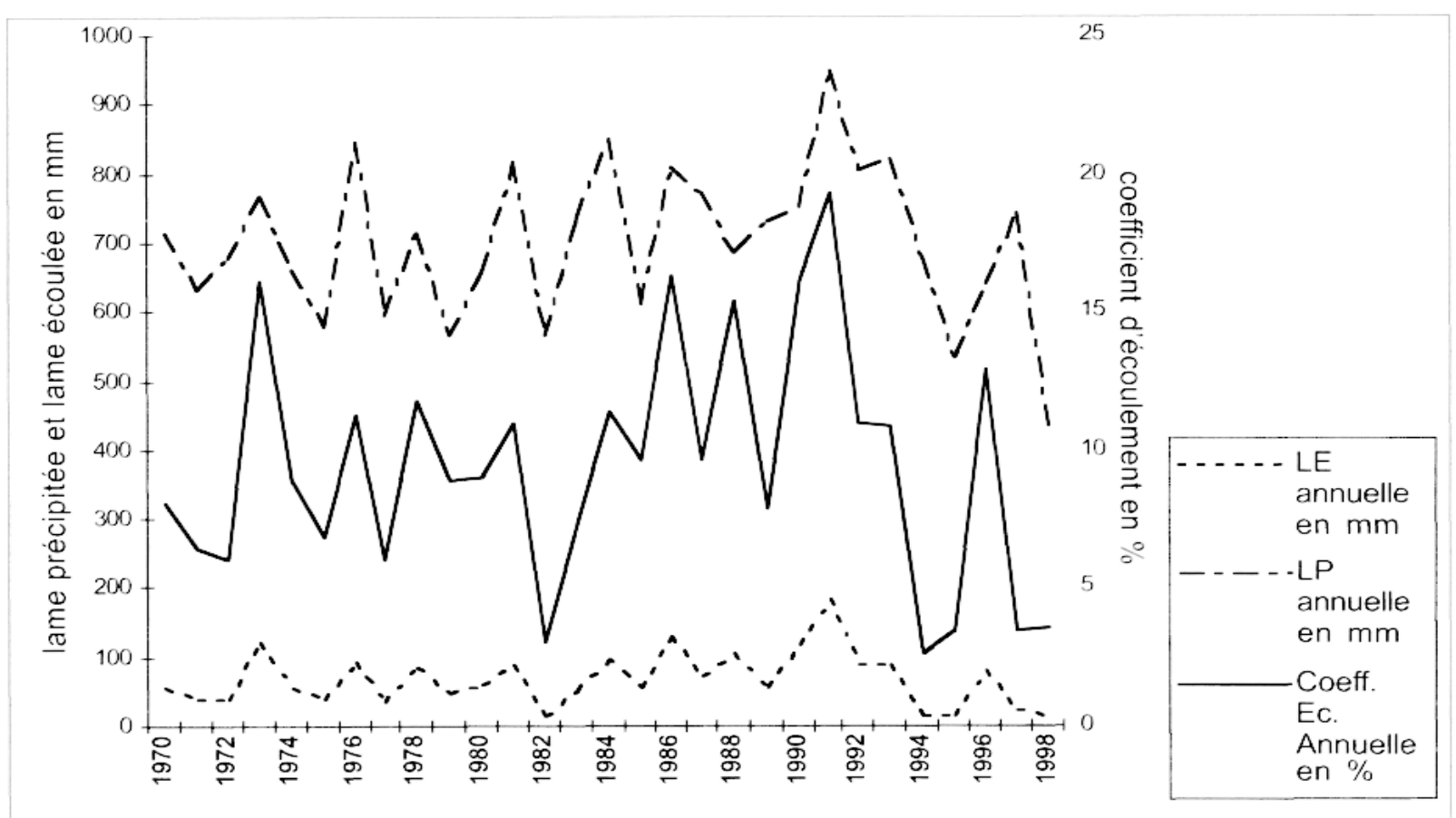

Fig. 2 : Évolution des lames précipitées et écoulées sur le bassin du Rio Ramos de 1970 à 1998

On n'a pas cherché ici à définir les indices d'écoulement de base, mais on a utilisé le même algorithme pour simplement séparer écoulements de base et de crue. I a méthode de Gustard et al. (1989) telle qu'utilisée par Humbert et Kaden (1994) peut se résumer comme suit :

À partir d'une chronique de débits journaliers, on divise la période de calcul en groupes de $\mathrm{N}$ jours non chevauchés ( $\mathrm{N}$ fixé à 3 dans notre cas). Recherche des minimums de chacun de groupes $(Q 1, Q 2, Q 3)$. Puis on scrute successivement tous les groupes $(Q 1$, Q2, Q3), (Q2, Q3, Q4), ( $\mathrm{n}-1, \mathrm{Q} n, \mathrm{Q}+1)$ pour trouver tous les point-pivots (QB1) de la courbe de débit de base; on répète l'opération sur tout le jeu de données pour obtenir tous les points-pivots $(\mathrm{QB2}, \mathrm{QB3} \ldots \mathrm{QBn})$.

Ensuite, par interpolation linéaire entre les $Q \mathrm{BB}$, on estime les valeurs journalières de QB. Puis on intègre les débits journaliers pour obtenir les volumes annuels d'écoulement de base (VB), d'écoulement de crue (VC), et d'écoulement total (V'I'). 
Afin de déterminer si les dégradations du milieu constatées ont pu modifier le comportement hydrologique de bassin-versants dans notre zone d'étude, on a utilisé deux longues séries de données (29) ans, de 1970 à 1998) de débits journaliers de deux stations de jaugeage : la station Salomé Acosta sur le Rio Sextin avec un bassin versant de 7128 km: et la station Sardinas sur le Rio Ramos avec un bassin versant de $4600 \mathrm{~km}$.

\section{RESLITATS}

I a décomposition des écoulements suivant l'algorithme de Ciustard permet donc d'obtenir pour chaque débit moyen journalier des séries des deux bassins concernés, un écoulement de base ( $Q$ base) et un écoulement de crue (Qcrue). I) cux exemples sont donnés dans les figures 3 et 4 .

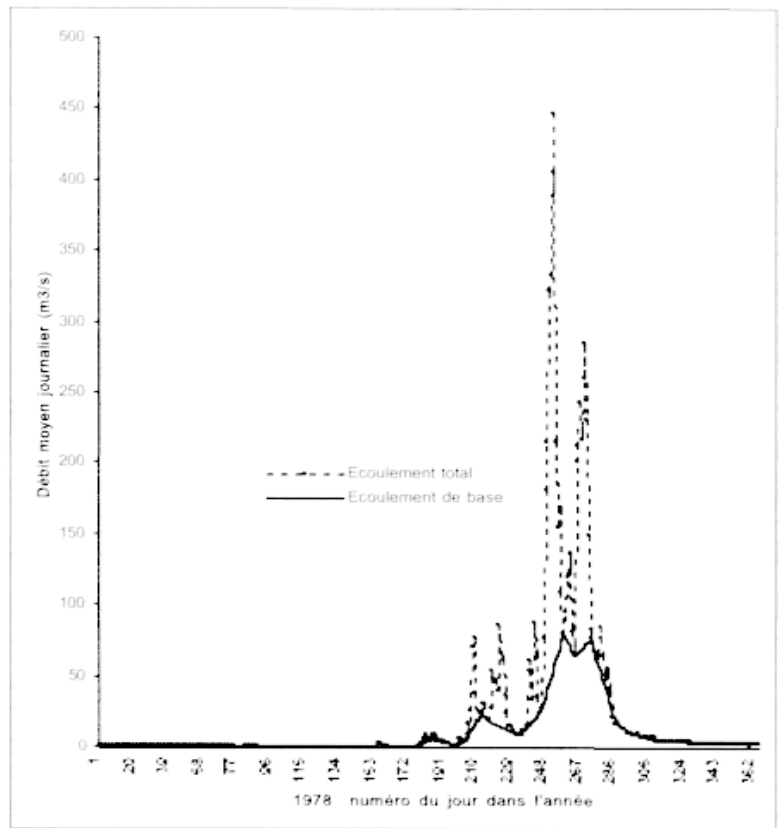

Fig. 3 : Décomposition de l'hydrogramme de l'année 1978. bassin du rio Ramos

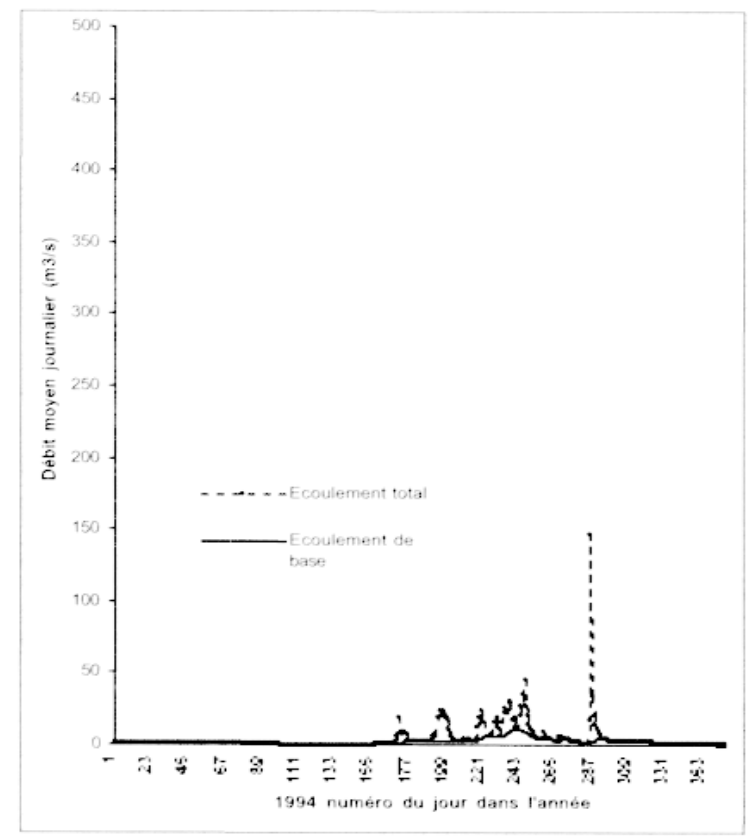

Fig. 4 : Décomposition de l'hydrogramme de l'année 1994, bassin du rio Ramos

( )n a utilisé ensuite le coefficient d'écoulement de base (Kbase) et celui de crue (Kcrue) dont la somme constitue le coefficient d'écoulement.

Unc régression entre les coefficients d'écoulement de base et de crue (Fï. 5), montre des coefficients de corrélation relativement bons et statistiquement significatifs (avec une probabilité de $95 \%$ ), mais surtout que la pente de la droite de corrélation augmente de décade en décade.

- entre 70-79 pour une valeur donnéc de Kbase, Kcrue $=2,302^{*} \mathrm{Kbase}$;

- entre 80-89, Kcruc $=2,685^{\circ}$ Kbase ;

- entre 90-98, Kcrue $=2,908^{*}$ Kbase.

Cela indique que la part du volume de base dans l'écoulement total annuel passe ainsi de $30 \%$ au cours des années 70 a moins de $25 \%$ au cours des années quatre-vingt-dix, 
ce qui dénote une augmentation appréciable du ruissellement de crue. Ceci traduit une "irrégularisation " des débits. La proportion d'eau ruisselant au moment de l'événement pluvieux augmente, la recharge des aquiferes locaux et régionaux est amoindrie, et avec elle la teneur en eau moyenne du sol, ainsi que sa capacité à restituer longtemps des débits de base aux cours d'eau. Enfin, des travaux en cours (Viramontes, 1999) ont montré que les temps de réponse des deux mêmes bassins ont diminué légèrement durant cette même période.
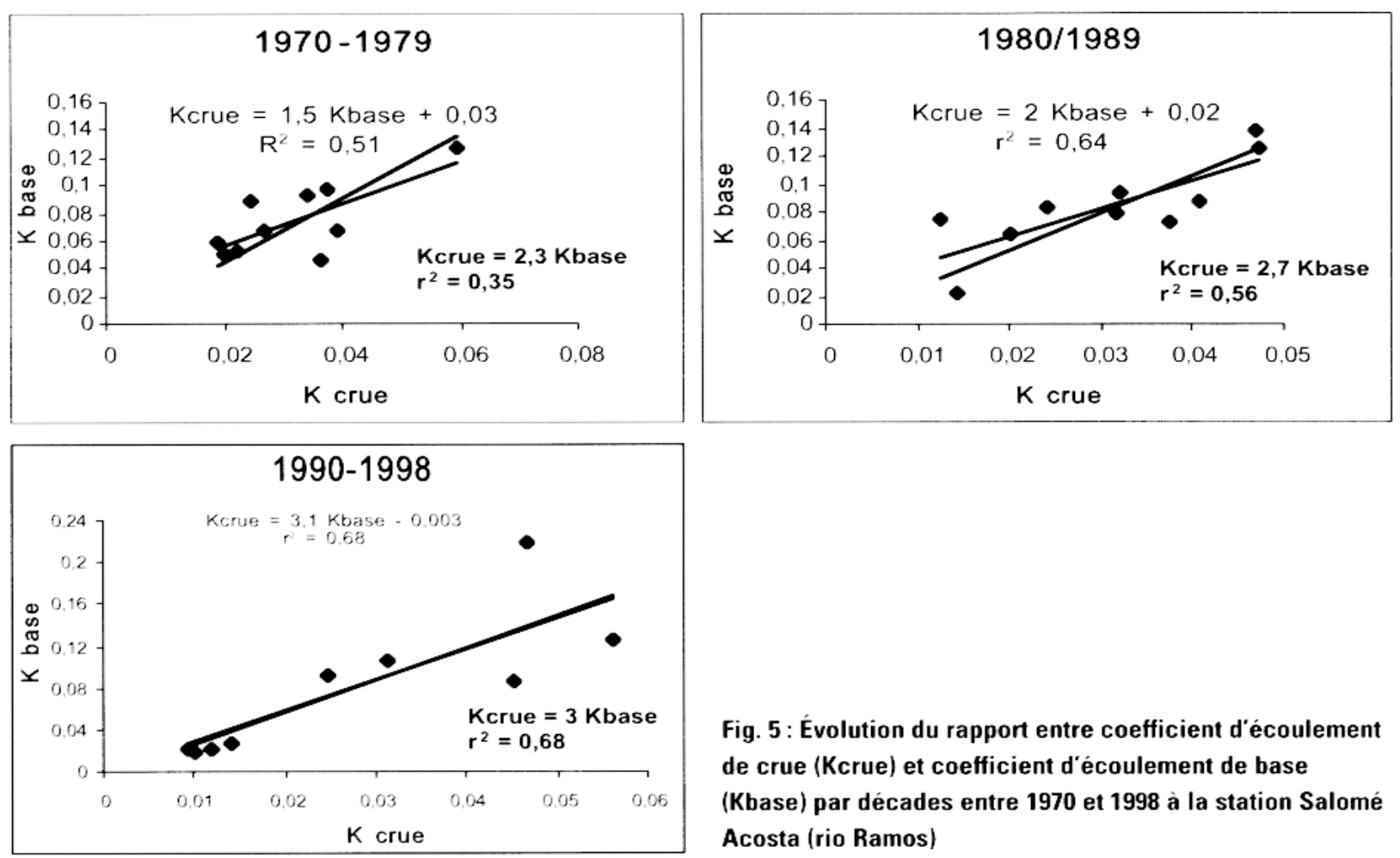

Fig. 5 : Évolution du rapport entre coefficient d'écoulement de crue (Kcrue) et coefficient d'écoulement de base (Kbase) par décades entre 1970 et 1998 à la station Salomé Acosta (rio Ramos)

\section{DISCUSSION}

Si l'on considère bien que les conditions climatiques n'ont pas changé, cette évolution pourrait bien être due à la dégradation du milieu ; ainsi, on a observé (ch. $2 c$ de la problématique) au niveau parcelle, des changements de comportements hydro-érosifs du fait de la dégradation du milieu (déboisement et surpâturage).

À l'échelle des deux grands bassins-versants (sur la fig. 5 celui du Ramos, le plus étendu des deux), l'accroissement de la part de l'écoulement de crue peut traduire une modification dans les conditions du ruissellement : la dégradation du réservoir naturel sol-végétation due à la surexploitation de l'espace a semble-t-il raccourci les temps de réponse des bassins, accentuant tant les crues que les étiages, phénomènes remarqués sous d'autres climats et à d'autres périodes, par exemple dans les Alpes Françaises du Sud, comme on peut le constater dans les archives (I)escroix, 1994). Cette même évolution a été remarquée aussi sur le bassin du rio Sextin. Ce genre de relations de cause à effet est éminemment géographique et pose au quotidien, surtout à l'heure actuelle 
dans les pays du Sud, le problème de l'adéquation de l'exploitation du milieu avec ses potentialités.

Étant donnée l'importance de la Sierra Madre pour l'approvisionnement en eau des régions arides environnantes, une modification des conditions du ruissellement et de l'érosion peut à terme entraîner de graves problèmes de développement. I.es risques possibles sont :

- un accroissement des transports solides et une accélération du colmatage du barrage de El Palmito, qui reçoit les eaux de tout le haut bassin du rio Nazas $\left(19000 \mathrm{~km}^{2}\right)$; ceci est valable pour tous les cours d'cau provenant de la Sicrra Madre, soit l'essentiel du territoire des États de Durango, Chihuahua, Sinaloa, Sonora (soit plus de $500000 \mathrm{~km}$ ' et 5 millions d'habitants) ; les mesures bathymétriques réalisées jusqu'à maintenant dans le barrage permettaient de prévoir une durée de vie du barrage de plusicurs siècles; mais qu'en serait-il en cas d'accélération drastique de l'érosion ;

- un accroissement de l'irrégularité interannuelle des écoulements des bassins versants qui pourra poser des problèmes dans la gestion de barrages tous destinés à l'irrigation ; pour la Nazas, le barrage de Palmito a été conçu pour assurer l'irrigation de l'ensemble de la Comarca Lagunera 9 années sur 10 ; or, durant la décennie 90, qui a commencé par l'année la plus pluvieuse depuis la mise en eau du barrage, le périmètre réellement irrigué a dû être réduit de $50 \%$ à 4 reprises ;

- à terme, la dégradation de l'espace pourrait se traduire par une évolution importante de l'évapotranspiration et du bilan total de l'eau; le risque d'être touché rapidement par le changement climatique global est sérieux.

\section{Conclusions}

Les premiers résultats nous ont montré que la pluviométric et le coefficient d'écoulement n'ont pas significativement changé. Les activités anthropiques sur le bilan hydrique régional n'ont pas modifié la quantité totale d'eau qui arrive à l'exutoire du bassin.

Par contre, une fois séparés les écoulements de base des écoulements de crue, on observe un changement de régime hydrique au niveau de la forme des hydrogrammes. En effet, le régime d'écoulement a évolué vers une diminution des temps de réponse et de concentration des caux dans le bassin versant : l'écoulement de base diminue petit à petit et les crues prennent une part croissante dans l'écoulement total.

Considérant que les changements climatiques des dernières 30 années ne sont pas significatifs (pas de tendance globale à l'augmentation ou a la baisse des précipitations), on pourrait dire que ces changements de comportement hydrique sont dus aux acrivités humaines (déforestation et surpâturage). 


\section{Bibliographie}

BARRAI. H. ct ANAYA E., 1995. - La ganadería y su manejo en relación con los recursos agua y pastizal en la zona semi-árida de México. Publication ORSTOM-INIFAP, Góme\% P'alacio, México, $\mathrm{n}^{\circ} 5,78 \mathrm{p}$.

BOSCH J.M. et HEWIEIT' J.D., 1982. - A review of catchment experiments to determine the effect of vegetation changes on water yield and evapotranspiration. J. of Hydr. 55 (1982) 3-23.

Boutrals J., 1994. - Eleveurs, bétail et environnement. À la croisée des parcours, ORSIOMCentre d'études africaines, pp. 303-319.

COSANDI:Y C., 1995. - La forêt réduit-elle l'écoulcment annuel? Annales de Géographie, n 581 582, pp. 7-25.

DIMANi A. et Robin C., 1975. - Las fases del vulcanismo en México ; una síntesis en relación con la evolución geodinámica desde el cretácico. Revista del Instituto de (icología, UNAM, México, $n^{\circ} 75(1)$, pp. $70-83$.

Drsc(ROIX L., 1994. - L'érosion actuclle dans la partic occidentale des Alpes du Sud. Thèse de Doctorat de géographic, Université Lyon II, 330 p.

Discrolx L. et Nouvilof J.F, 1997. - Escurrimiento y erosión en la Sicra Madre Occidental. Publication ORSTOM-INIYAP, (jómez Palacio, México, 50 p.

FRTTSCH J.-M., 1990. - I es effets du défrichement de la forêt amazonienne et de la mise en culture sur l'hydrologie de petits bassins-versants en Guyane française. Thèse, USTL, Montpellier, nov. $1990,390 \mathrm{p}$.

(il:NTRY H.S., 1957. - I.os pastizales de Durango. Fstudio ecológico, fisiográfico y florístico. IMRNR México.

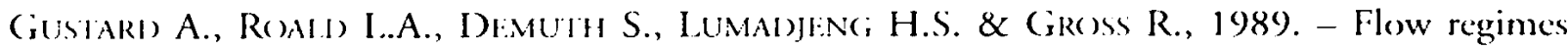
from experimental and network data (FREND). Institute of Hydrology, Wallingford (UK), 2 vol.

H(MBBtRI J. et KAI)IN U., 1994. - Détection des modifications de l'écoulcment fluvial au moyen de l'indice de débit de base. Revue de Géographie Alpine, 82, n² 2, pp. 25-36.

Me:Dowent. F.W. et C1.Abaugin S.F., 1979. - Ignimbrites of the Sierra Madre Occidental and their relation to the tectonic history of western Mexico. Geological Society of America bulletin. Special paper 180.

Notivliof J.F et Drscirolx I.., 1996. - Aridité et sécheresses du Nord-Mexique. CEMCA, Mexico, Trace, $n^{\circ} 30$, pp. 9-25.

P(OIIINARI) J., DI.SC KOIX L. et JANI:AC J.L., 1995. - Surpâturage et formation de terrassettes sur les versants de la Sicrra Madre Occidentale (Nord-Ouest de Mexique), Revue de Géographie Alpine, $n^{\circ} 2$, pp. 77-86,

RoI)RI(;UF\% M.G., 1997. - Determinación de la vegetación en la Sicrra Madre Occidental para la calibración de imágenes satćlite. ECF-UJED, Durango, México, 60 p.

SI:RRATI C., 1978. - Dynamique des versants de Haute Montagne: Andes Péruviennes - Alpes Briançonnaises. 'Thèse de géographic, Université Paris VII, 320 p.

VIRAMONI D. D., 1995. - Caracterización de los suelos y la vegetación en la parte alta de la cuenca del Nazas. Publication ORSTOM-INIFAP, Gómez Palacio, México, 42 p.

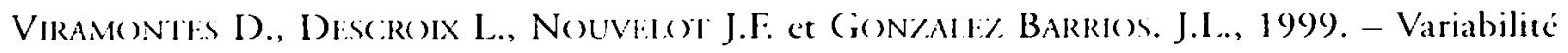
des apports liquides et solides dans un grand bassin endoréique du Nord Mexique : le bassin Nazas-Aguanaval $(92000 \mathrm{~km}$ ). International Symposium " Hydrological and (ieochemical Processes in large scale river basins". Hibam-IRI), Manaus, nov. 99. 
Résumé : La Sicrra Madre Occidentale (Nord-Ouest du Mexique) est un véritable château d'eau pour les zones de plaine avoisinantes, semi-arides et arides, qui ont profité des écoulements pour établir des zones agricoles importantes (c'est le cas de la Laguna avec plus d'un million d'habitants). Cependant, dans cette chaine montagneuse, la surexploitation des ressources naturelles par l'élevage et l'exploitation forestière ont eu comme conséquences une dégradation du milieu. Pour mettre en évidence les effets hydriques de ces changements, on a tenté de décomposer les hydrogrammes des débits journaliers des cours d'cau.

À partir de l'Indice de l'Écoulement de Base (IF.B), on essaic d'interpréter les conséquences hydrologiques de ces changements progressifs du milieu.

Les résultats nous montrent des tendances de changement du comportement de régime hydrique. Ce n'est pas le volume total d'eau écoulće qui a changé, mais la façon dont l'écoulement se présente. Avec des débits plus irréguliers dans les dernières années, la relation entre écoulement de crue et écoulement total devient de plus en plus marquée. l'écoulement de base diminue petit à petit et les crues prennent la place de la majorité de l'écoulement total, ce qui pourrait être une conséquence du surpâturage et du déboisement.

Mots-clés : écoulement de base, déboisement, surpâturage, Nord-Mexique

Resumen : Ia Sierra Madre Occidental (Noroeste de México) es un tanque natural de aguas para las llanuras colindantes, semiáridas $y$ áridas, que han aprovechado sus escurrimientos para establecer importantes perímetros agrícolas (entre cllos la Comarca Laguncra, con más de un millón de habitanres). Sin cmbargo, en esta sierra, la sobreexplotación de los recursos naturales por la ganadería y la explotación forestal tuvicron como consecuencia una degradación del Medio Ambiente. Para determinar los efectos hídricos de estos cambios, se intentó descomponer los hidrogramas de gastos diarios de los ríos principales.
À partir del Indice de Escurrimiento Base (IEB), se intenta interpretar las consecuencias hidrológicas de estos cambios progresivos del medio.

Los resultados enseñan tendencias de cambio del comportamiento del régimen hídrico. El volumen total de aguas escurridas anualmente no es el que cambia, sino la manera en que se presenta el escurrimineto. Con gastos más irregulares en los últimos años, la relación entre escurrimiento directo y escurrimiento total se vuclve cada vez más evidente. El escurrimiento base disminuye poco a poco y las avenidas ocupan una proporción superior del escurrimiento total, lo que podría ser una consecuencia del sobrepastoreo y del desmonte.

Palabras claves: Indice de Escurrimiento Base, desmonte, sobrepastoreo, Norte de México

Abstract: The western Sierra Madre represents a "water tank" for surrounding plains and plateaux that have an arid or semi arid climate; these regions have used during more than fifty years overland flow to practice irrigation in great areas (for example the Laguna with 160000 ha has more than 12 million inhabitants). However, in the mountain, overexploitation of natural resources by breeding and forestry results in an environment degradation. A decomposition of hydrograms has been attempted to put in light eventual changes in water balance. Using an algorithm which allows to separate base flow and flood flow, an interpretation of hydrological trend of hydrologic behaviour of the basins. The annual runoff does not change; but the way runoff occurs has changed. Discharges are mopre irregular in the last decades; the proporrion of base flow in annual discharge decreases and inversely, flood flow enhances. This is what appears as the conseguences of the landscape degradation : overgrazing and deforestation have accelarated runoff. The hydrological evolution leads environmental and economical consequences to downstrean arid zones, which absolutely need the water of the western Sicrra Madre.

Keywords: base flow, deforestation, overgrazing. Northern Mexico 


\begin{abstract}
Abstract : The western Sierra Madré represents a "water tank" for surrrounding plains and plateaux that have an arid or semi arid climate; these regions have used during more than fifty years overland flow to practice irrigation in great areas (for example the Laguna with 160000 ha has more than 12 million inhabitants). However, in the mountain, ove- rexploitation of natural resources by breeding and forestry results in an environment degradation. A decomposition of hydrograms has been attempted to put in light eventual changes in water balance. Using an algorithm which allows to separate base flow and flood flow, an interpretation of hydrological trend of hydrologie behaviour of the basins. The annual runoff does not change; but the way runoff occurs has changed. Discharges are topre irregular in the last decades; the proportion of base flow in annual discharge decreases and inversely, flood flow enhances. This is what appears as the consequences of the landscape degradation : overgrazing and deforestation have accelarated runoff. The hydrological evolution leads environmental and economical consequences to downstrean arid zones, which absolutely need the water of the western Sierra Madre.
\end{abstract}

\title{
Résumé
}

Résumé : La Sierra Madré Occidentale (Nord-Ouest du Mexique) est un véritable château d'eau pour les zones de plaine avoisi- nantes, semi-arides et arides, qui ont profité des écoulements pour établir des zones agricoles importantes (c'est le cas de la Laguna avec plus d'un million d'habitants). Cependant, dans cette chaîne montagneuse, la surexploitation des ressources naturelles par l'élevage et l'exploitation forestière ont eu comme conséquences une dégradation du milieu. Pour mettre en évidence les effets hydriques de ces changements, on a tenté de décomposer les hydrogrammes des débits journaliers des cours d'eau. À partir de l'Indice de l'Écoulement de Base (IEB), on essaie d'interpréter les conséquences hydrologiques de ces changements progressifs du milieu. Les résultats nous montrent des tendances de changement du comportement de régime hydrique. Ce n'est pas le volume total d'eau écoulée qui a changé, mais la façon dont l'écoulement se présente. Avec des débits plus irréguliers dans les dernières années, la relation entre écoulement de crue et écoulement total devient de plus en plus marquée. L'écoulement de base diminue petit à petit et les crues prennent la place de la majorité de l'écoulement total, ce qui pourrait être une conséquence du surpâturage et du déboisement.

\section{Resumen}

Resumen : La Sierra Madré Occidental (No- roeste de Mexico) es un tanque natural de aguas para las llanuras colindantes, semiáridas y áridas, que han aprovechado sus escurri- mientos para establecer importantes perime- tros agricolas (entre ellos la Comarca Lagunera, con más de un milión de habitantes). Sin embargo, en esta sierra, la sobreex- plotación de los recursos naturales por la ganaderia y la explotación forestal tuvieron como consecuencia una degradación del Medio Ambiente. Para determinar los efectos hidricos de estos cambios, se intentó descom- poner los hidrogramas de gastos diarios de los ríos principales. À partir del Indice de Escurrimiento Base (IEB), se intenta interpretar las consecuencias hidrológicas de estos cambios progresivos del medio. Los resultados ensefian tendencias de cambio del comportamiento del regimen hidrico. El volumen total de aguas escurridas anualmente no es el que cambia, sino la manera en que se présenta el escurrimineto. Con gastos más irregulares en los últimos afios, la relación entre escurrimiento directo y escurrimiento total se vuelve cada vez más évidente. El escurrimiento base disminuye poco a poco y las avenidas ocupan una proporción superior del escurrimiento total, lo que podría ser una consecuencia del sobrepastoreo y del des-

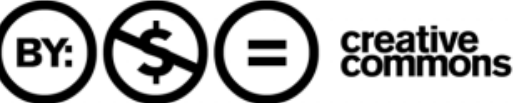

